\title{
Optimal Planning of Solar-Diesel Generation System with Energy Storage
}

\author{
Vladimir A. Tremyasov*, \\ Yana E. Zograf and Tatyana V. Krivenko \\ Siberian Federal University \\ Krasnoyarsk, Russian Federation
}

Received 18.12.2020, received in revised form 29.01.2021, accepted 14.02.2021

\begin{abstract}
For power supply to isolated consumers, an autonomous power complex is used, consisting of photovoltaic converters, diesel generators and storage batteries. When planning the power complex, the fundamental task is to select the optimal structure of the elements of the generation system, which maximizes the use of the solar energy input to ensure the given consumer load. The article proposes a technique for optimizing a swarm of particles in relation to the problem of determining the composition and power of the elements of a solar-diesel generation system. On the example of the power supply system of the settlement of the Republic of Khakassia, the optimal structure of generating and storage facilities using solar energy has been determined. To assess the reliability indicators of the power complex, a probabilistic method has been proposed and implemented, which makes it possible to take into account the daily cyclicity and seasonal variability of the intensity of solar radiation.
\end{abstract}

Keywords: isolated consumer, solar-diesel generation system, diesel generator, photovoltaic panels, storage batteries, particle swarm optimization, reliability.

Citation: Tremyasov V.A., Zograf Ya.E., Krivenko T.V. Optimal planning of solar-diesel generation system with energy storage, J. Sib. Fed. Univ. Eng. \& Technol., 2021, 14(1), 42-54. DOI: 10.17516/1999-494X-0287

(C) Siberian Federal University. All rights reserved

This work is licensed under a Creative Commons Attribution-Non Commercial 4.0 International License (CC BY-NC 4.0).

* Corresponding author E-mail address: ezhaky@mail.ru 


\title{
Оптимальное планирование
}

\author{
солнечно-дизельной системы генерации \\ с аккумулированием электроэнергии
}

\author{
В.А. Тремясов, Я. Е. Зограф, Т. В. Кривенко \\ Сибирский федеральный университет \\ Российская Федерачия, Красноярск
}

\begin{abstract}
Аннотация. Для электроснабжения изолированных потребителей используется автономный энергокомплекс, состоящий из фотоэлектрических преобразователей, дизельных генераторов и аккумуляторных батарей. При планировании энергокомплекса основополагающей задачей является выбор оптимальной структуры элементов системы генерации, при которой максимально используется поступление солнечной энергии для обеспечения заданной нагрузки потребителя. В статье предложена методика оптимизации роя частиц применительно к задаче определения состава и мощности элементов солнечно-дизельной системы генерации. На примере системы электроснабжения населенного пункта Республики Хакасия определена оптимальная структура генерирующих и аккумулирующих установок с использованием солнечной энергии. Для оценки показателей надежности энергокомплекса предложен и реализован вероятностный метод, позволяющий учитывать суточную цикличность и сезонную изменчивость интенсивности солнечного излучения.
\end{abstract}

Ключевые слова: изолированный потребитель, солнечно-дизельная система генерации, дизельный генератор, фотоэлектрические панели, аккумуляторные батареи, оптимизация роя частиц, надежность.

Цитирование: Тремясов, В.А. Оптимальное планирование солнечно-дизельной системы генерации с аккумулированием электроэнергии / В.А. Тремясов, Я.Е. Зограф, Т.В. Кривенко // Журн. Сиб. федер. ун-та. Техника и технологии, 2021, 14(1). C. 42-54. DOI: 10.17516/1999-494X-0287

\section{Введение}

При планировании и проектировании автономной солнечно-дизельной системы генерации (СДСГ) основными задачами являются: выбор оптимальной структуры генерирующих элементов, при котором эффективно используется поступление солнечной энергии для покрытия нагрузки изолированного потребителя, и анализ надежности функционирования энергокомплекса.

Применение фотоэлектрических преобразователей (ФЭП) с накопителями энергии - аккумуляторными батареями (АБ) совместно с традиционными дизель-генераторами (ДГ) позволит снизить топливную составляющую дизельных электростанций (ДЭС) в себестоимости вырабатываемой электроэнергии, что существенно повысит технико-экономическую эффективность СДСГ.

Состояние системы генерации, включая типы генераторов, их размещение, мощности, обеспечение ее надежности в будущем, является результатом планирования системы генерации, которое представляет сложную задачу. В настоящее время моделированию СДСГ и оптимальному выбору структуры автономного энергокомплекса с использованием солнечной энергии посвящено большое количество работ как в России $[1,2]$, так и за рубежом $[3,4]$. 
Также существует широкий спектр компьютерных программ, предназначенных для решения оптимизационных задач возобновляемой энергетики, среди которых, как наиболее популярные, можно выделить следующие:

- Hybrid optimization by genesis algorithm (HOGA, Испания) [5],

- Hybrid optimization modeling software (HOMER, США) [6],

- Hybrid power optimized for rural/remote areas (HYPORA, CША) [7].

Однако в указанных работах при выборе генерирующих элементов автономного энергокомплекса с использованием солнечной энергии не в полной мере учитываются изменения графика нагрузки потребителей, выработка мощности ФЭП (суточная, месячная, годовая) в зависимости от актинометрических и метеорологических условий местности, технических характеристик и способа ориентации ФЭП. Поэтому необходимо совершенствование методик оптимизации структуры автономного энергетического комплекса ФЭП-АБ-ДГ с учетом вышеперечисленных факторов, методов оценки надежности и их реализации в программновычислительном комплексе.

Основную цель работы можно обозначить следующим образом: оптимальное проектирование и планирование системы генерации на основе сочетания фотоэлектрических преобразователей, дизельных генераторов и накопителей электроэнергии с учетом различных технологий использования, анализа их надежности и экономических характеристик.

\section{Методика оптимизации структуры СДСГ}

Для решения поставленной задачи оптимизации как одна из наиболее эффективных была выбрана методика оптимизации роя частиц.

Оптимизация роя частиц основана на моделировании социального поведения роя, например, такого, как стая птиц. При оптимизации роя частиц каждая частица пытается найти свою наилучшую позицию (решение) в многомерном пространстве (размерности переменных управления) [8]. Во время полета каждая частица корректирует свою позицию по ее текущей скорости, ее собственному предыдущему опыту и опыту других частиц. Процесс постепенно достигает лучшей позиции (оптимального решения). Частицы в терминах используемой методики представляются набором координат в пространстве решений и, соответственно, связанным с ними решением. Оптимизация роя частиц - это методика вероятностного поиска, которая перемещает позиции от одного к другому набору решений.

Подход с инерцией массы основан на следующем правиле обновления [9]:

$$
\begin{aligned}
& V_{i k}^{(t+1)}=w V_{i k}^{(t)}+\varphi_{1} \cdot \alpha_{1}^{(t)} \cdot\left(P b_{i k}^{(t)}-x_{i k}^{(t)}\right)+\varphi_{2} \cdot \alpha_{2}^{(t)} \cdot\left(G b_{k}^{(t)}-x_{i k}^{(t)}\right), \\
& x_{i k}^{(t+1)}=x_{i k}^{(t)}+V_{i k}^{(t+1)} .
\end{aligned}
$$

Здесь $V_{i k}$ и $x_{i k}$ - соответственно скорость и позиция $k$-й переменной $i$-й частицы; $P b_{i k}-$ лучшая позиция $k$-й переменной $i$-й частицы, где «лучшая» подразумевает, что эта позиция приводит к лучшему значению целевой функции; $G b_{k}$-лучшая глобальная позиция $k$-й переменной во всех частицах; $\varphi_{1}$ и $\varphi_{2}$ - два коэффициента ускорения; $\alpha_{1}$ и $\alpha_{2}$ - два однородно распределенных случайных числа в диапазоне $[0,1] ; w$ - коэффициент утяжеления инерции, который 
отражает эффект скорости в строго предыдущей итерации; верхний индекс $(t)$ или $(t+1)$ представляет значение итерации.

Можно заметить, что сущность оптимизации роя частиц состоит в том, чтобы вычислить новую скорость каждой переменной всех частиц, которые рассматриваются, используя ее текущую скорость, расстояние между ее текущим положением и его собственной предыдущей лучшей позицией и расстояние между его текущим положением и предыдущей лучшей позицией той же самой переменной среди других частиц в рое. Эти два расстояния случайно взвешены в обновлении скорости. Как только вычислена новая скорость, обновляется новая позиция перемещением в шаг при новой скорости.

Эти три параметра $w, \varphi_{1}$ и $\varphi_{2}$ воздействуют на скорость сходимости. Если значения $\varphi_{1}$ и $\varphi_{2}$ слишком маленькие, то процесс оптимизации будет медленным, потому что потребуется относительно большое количество итераций. Однако чрезмерно большие значения $\varphi_{1}$ и $\varphi_{2}$ могут вызвать неустойчивость в числовых вычислениях. Соответствующие значения этих двух параметров различны для различных проблем, и нет твердого правила для того, чтобы их выбрать. Чтобы определить их значения, необходимо испытание. Фактор инерции массы $w$ часто определяется как реальное значение в интервале $[0,0 ; 1,0]$ и может быть пропорционально уменьшен с итерацией

$$
w=w_{\max }-\frac{t}{t_{\max }}\left(w_{\max }-w_{\min }\right),
$$

где $w_{\max }$ и $w_{\min }$ - максимальные и минимальные значения массы; $t u t_{\max }$ - текущий и максимальный номер итерации соответственно.

Целевой функцией в методике оптимизации роя частиц является комбинированная себестоимость электроэнергии, полученной от СДСГ, определяемая по выражению, руб/кВт·ч,

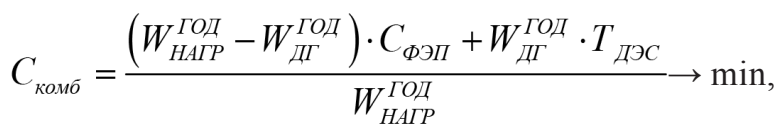

где $W_{H A Г P}^{\Gamma о Д}-$ потребление электроэнергии нагрузкой; $W_{\text {дІ }}^{\text {ГоД }}$ - выработка электроэнергии ДГ;

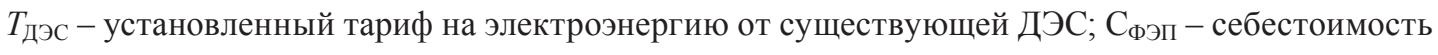
электрической энергии, производимой от ФЭП, руб/кВт·ч.

$$
C_{\Phi Э I}=\frac{3+И \cdot T_{O K}}{W_{\Phi \ni I I}^{\Gamma О Д} \cdot T_{O K}},
$$

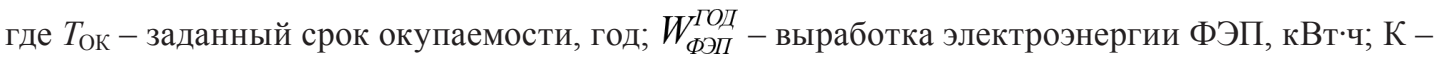
капитальные затраты на строительство энергокомплекса ФЭП-АБ-ДГ; И - ежегодные издержки на эксплуатацию ФЭП-АБ-ДГ.

Для повышения качества оптимизационных решений предлагается использование дневных графиков нагрузки, усредненных за месяц, что позволяет рассчитать потребность в электроэнергии с большей точностью в сравнении с усреднением за год. Также используются зависимости, отражающие влияние температуры окружающей среды на выработку электроэнергии ФЭП, приведенные в [10].

Зная количество и мощность генерирующих и аккумулирующих элементов, можно определить вклад каждого в общую выработку электроэнергии, руководствуясь следующими правилами: 
А. Если энергия, вырабатываемая ФЭП, больше, чем требуется для покрытия нагрузки, ее излишки уходят на заряд АБ.

Б. Если энергии от ФЭП недостаточно для покрытия нагрузки, но имеется достаточный заряд АБ, дефицит покрывается за счет АБ. Важно отметить, что заряд батарей не должен падать ниже $30 \%$ от их номинальной емкости.

В. Если емкости АБ недостаточно для полного покрытия дефицита, но их заряд выше $30 \%$, происходит разряд АБ до порогового значения, а затем для питания нагрузки подключаются ДГ.

Г. Если батареи разряжены, весь дефицит покрывается выработкой электроэнергии ДГ.

Процедура использования методики исследования роя частиц для решения задачи оптимизации при планировании СДСГ включает следующие шаги:

1. Переменные управления в первичной проблеме оптимизации являются переменными

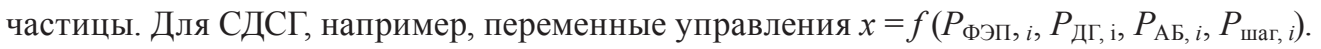

2. $N$ начальных частиц (т. е. $N$ выполнимых, но не оптимальных решений) создаются случайным образом. Каждый из $N$ субъектов получен следующим образом: каждая переменная управления находится по выражению

$$
x_{k}=r_{k}\left(x_{k \max }-x_{k \min }\right)+x_{k \min },
$$

где $x_{k}-k$-я переменная; $x_{k \max }$ и $x_{k \min }-$ верхняя и нижняя границы $x_{k} ; r_{k}$ - однородно распределенное случайное число в интервале $[0,1]$ для $k$-й переменной. Каждая переменная имеет независимое $r_{k}$. Если переменная - целое число, то выражение $r_{k}\left(x_{k \max }-x_{k \text { min }}\right)$ должно быть округлено до целого числа.

Так же случайно созданы $N$ скоростей с использованием подобного подхода.

3. Вычисляются значения целевой функции, полученные из обновления каждой переменной для каждой частицы. «Лучшая позиция» приписывается той, которая приводит к минимуму целевой функции. Начальная лучшая позиция $P b_{i k}$ каждой переменной в каждой частице ее начальная позиция в первой итерации. Лучшая глобальная позиция $G b_{i k}$ каждой переменной является позицией, соответствующей минимуму в значениях целевой функции, полученных из обновления той же переменной в $N$ частицах, т. е. в рое.

4. Скорости каждой переменной каждой частицы обновляются по уравнению (1).

5. Позиции каждой переменной каждой частицы обновляются по уравнению (2). Вычисляются себестоимости электроэнергии в СДСГ для текущих позиций всех частиц.

6. Обновляется лучшая позиция каждой переменной каждой частицы и лучшие глобальные позиции каждой переменной для всех частиц.

7. Если у значений оптимизационной функции нет существенного изменения в нескольких последовательных итерациях или если итеративный процесс достигает заданного максимального числа итераций, оптимизация заканчивается. Иначе переход на шаг 4.

С каждой точкой (частицей) из пространства решений связано значение целевой функции, вычисляемое при переходе к ее координатам. Переменными при оптимизации выступают модели дизельных ДГ, ФЭП, АБ и значение установленной мощности ФЭП. Шаг варьирования установленной мощности ФЭП выступает как ограничение и задается в виде 


$$
P_{\text {наг }, i}=k P_{\text {наг }}
$$

где $k=(0, \ldots, 1)$, с шагом 0,$15 ; P_{\text {НАг }}-$ мощность нагрузки.

Вторым ограничением служит условие, согласно которому количество ДГ не может быть меньше двух из соображений надежности.

Расчет целевой функции включает в себя определение количества элементов системы ФЭП-ДГ-АБ, распределение нагрузки между ними и вычисление себестоимости электроэнергии, вырабатываемой рассматриваемой конфигурацией.

Разработана компьютерная программа в среде Matlab, реализующая описанную методику, которая позволяет выполнить поиск оптимального решения.

Оптимизацию СДСГ рассмотрим на примере населенного пункта в Хакасии, где установлена ДЭС мощностью 200 кВт, обеспечивающая выработку 388 МВт·ч/год. Стоимость вырабатываемой электроэнергии составляет 60 руб/кВт. В селе проживет около 1500 человек. Подача электроэнергии от дизельных генераторов происходит по расписанию: с 5 до 11 ч и с 17 до 23 ч зимой; с 6 до 11 ч и с 18 до 23 ч в летний период.

При построении автономной системы электроснабжения предлагается использовать СДСГ с аккумуляторами без промежуточных шин (рис. 1).

Для рассматриваемого поселка получены типовые суточные графики нагрузки в зимний и летний период (рис. 2).

В результате оптимизации были выбраны: поликристаллические солнечные панели модели «SIP300-5BB» - 600 шт.; аккумуляторные батареи модели «MLQ12-150» - 401 шт.; дизельные генераторы «АД100-Т400»- 2 шт. Капитальные затраты составили 23,884 млн руб. Себестоимость производимой СДСГ электроэнергии $-35,47$ руб/кВт·ч.

На рис. 3 представлен интерфейс программы, в правой нижней части которого выведены результаты оптимизации рассмотренной СДСГ.

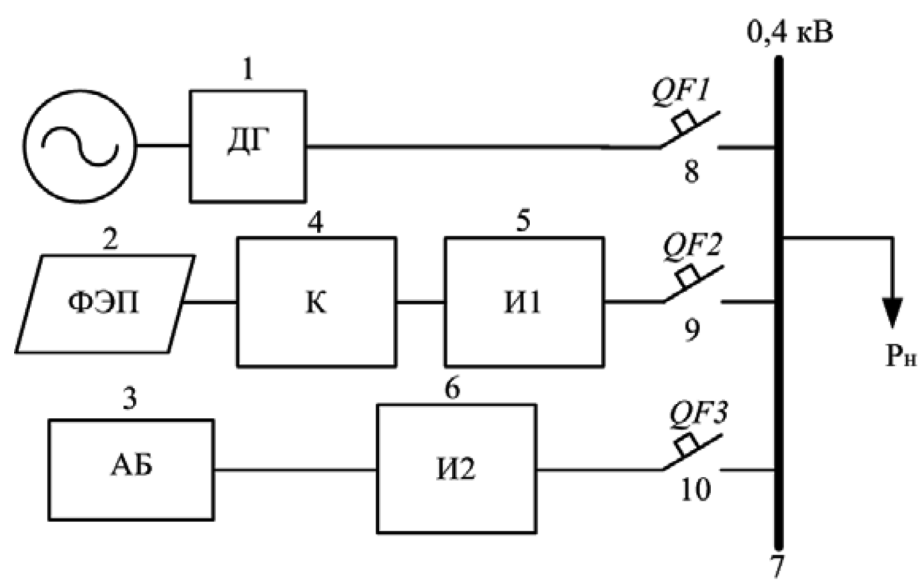

Рис. 1. Автономная солнечно-дизельная система генерации в населенном пункте Хакасии: 1 - дизельгенератор; 2 - фотоэлектрические панели; 3 - аккумуляторная батарея; 4 - конвертор; 5, 6 - инвертор; 8, 9,10 - автоматический выключатель

Fig. 1. Autonomous solar-diesel generation system in the settlement of Khakassia: 1 - diesel generator; 2 - photovoltaic panels; 3 - storage battery; 4 - converter; 5, 6 - inverter; $8,9,10$ - automatic breaker 


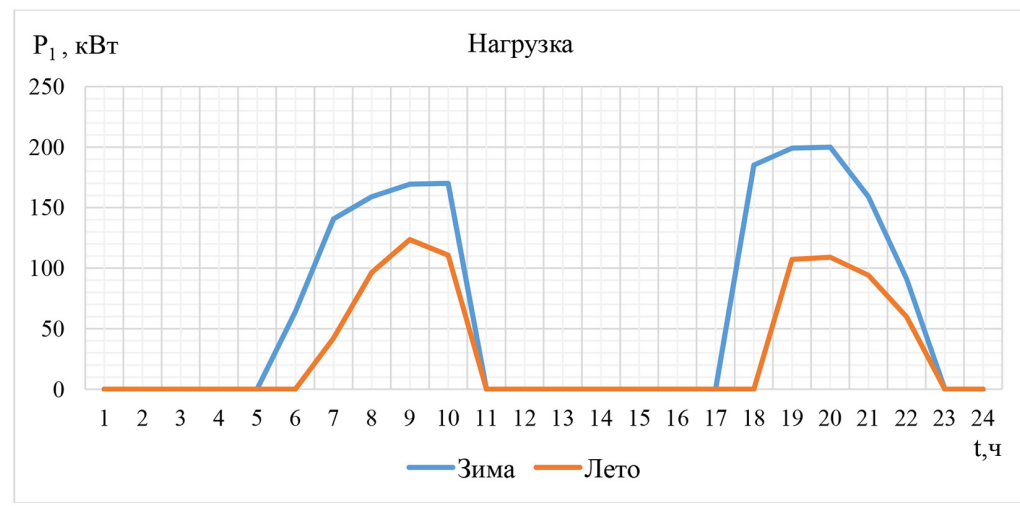

Рис. 2. Суточные графики нагрузки села

Fig.2. Daily load graphs of the village

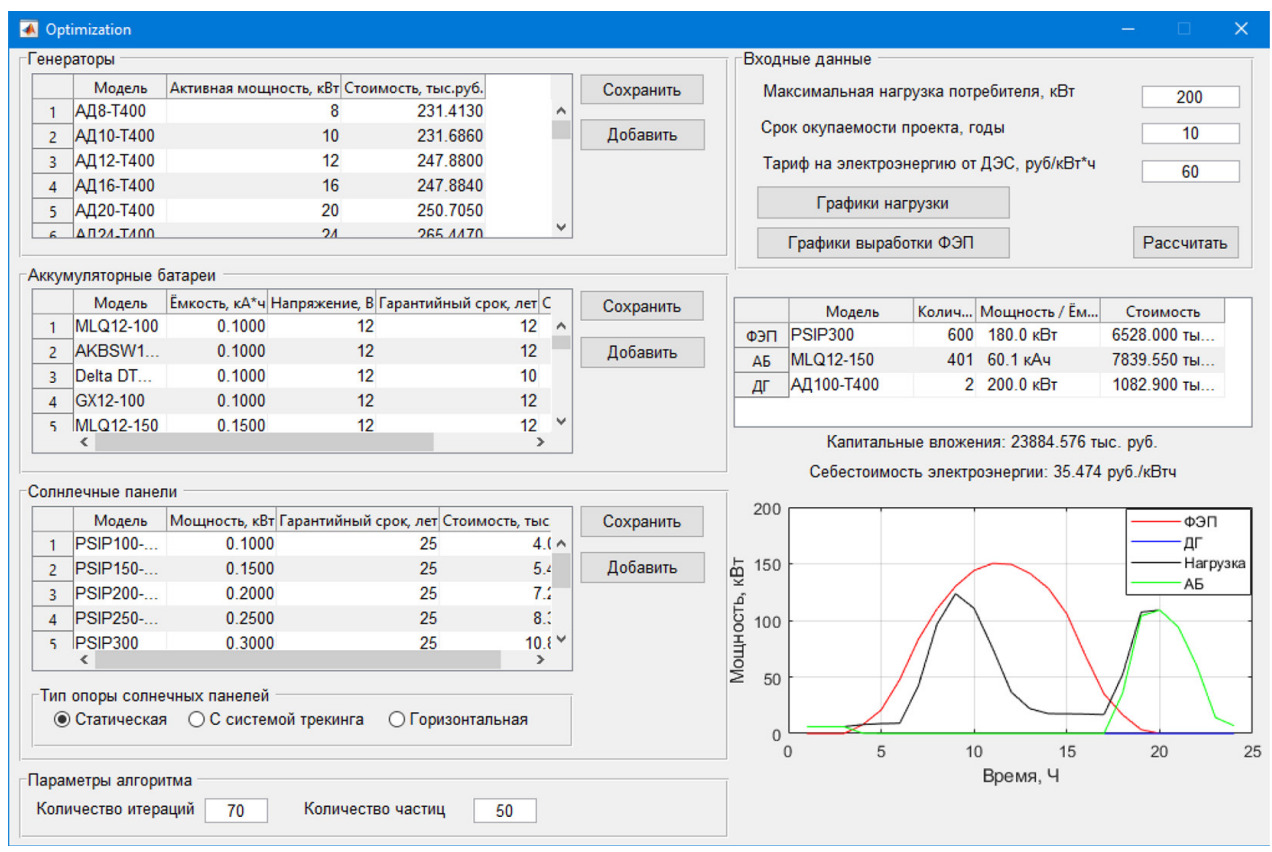

Рис. 3. Интерфейс компьютерной программы

Fig. 3. Computer program interface

На рис. 4, 5 показано оптимальное распределение мощности между генерирующими элементами и АБ при наклонном положении ФЭП. Очевидно, что снижение себестоимости электроэнергии большей частью достигается за счет установки ФЭП и АБ в количестве, позволяющем в благоприятные периоды солнечной активности полностью отключать ДГ. Также это отражено на графике в окне программы (рис. 3).

В табл. 1 представлено сравнение себестоимости электроэнергии при различных сроках окупаемости, для электростанции с наклонным расположением фотоэлектрических панелей и при тарифе на электроэнергию от ДЭС в 60 руб/кВт·ч. 
Таблица 1. Изменение себестоимости при различных сроках окупаемости проекта

Table 1. Change in cost for different payback periods of the project

\begin{tabular}{|l|c|c|c|c|c|c|c|}
\hline $\begin{array}{l}\text { Срок окупаемости, } \\
\text { год }\end{array}$ & 5 & 8 & 10 & 12 & 15 & 17 & 20 \\
\hline $\begin{array}{l}\text { Себестоимость, руб/ } \\
\text { кВт·ч. }\end{array}$ & 42,74 & 37,35 & 35,47 & 34,22 & 32,97 & 32,38 & 31,72 \\
\hline
\end{tabular}

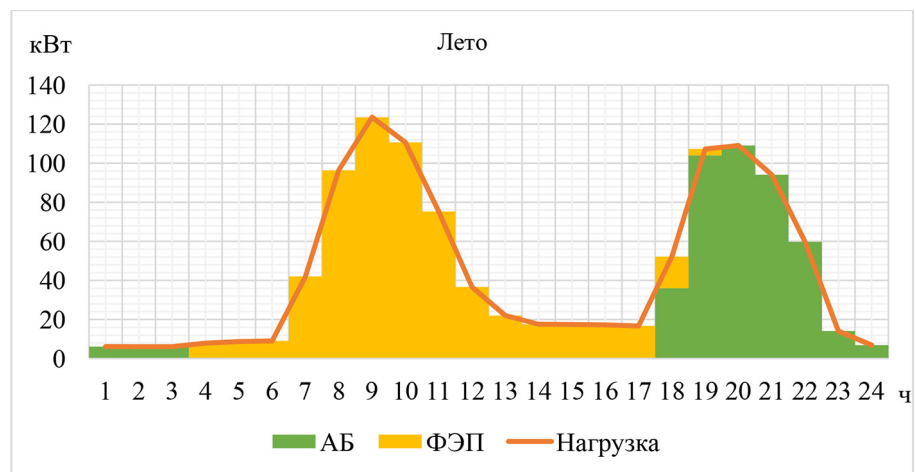

Рис. 4. Распределение мощности между элементами СДСГ в летний период

Fig. 4. Power distribution between SDSG elements in summer

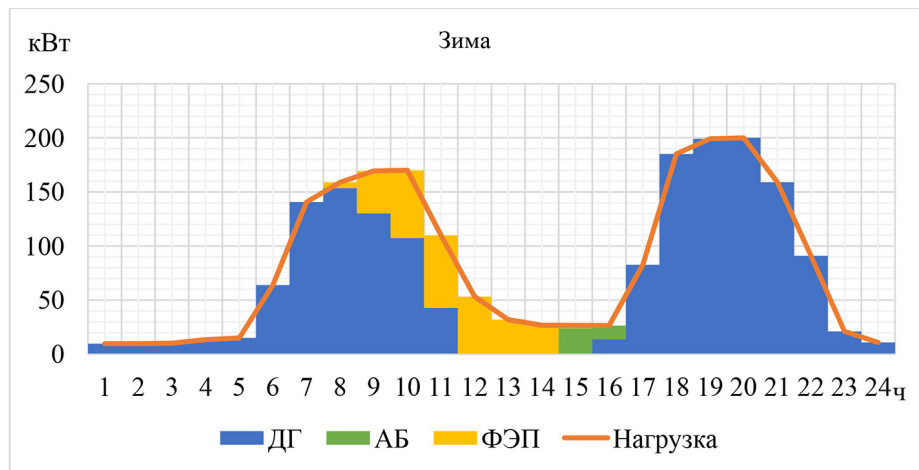

Рис. 5. Распределение мощности между элементами СДСГ в зимний период

Fig. 5. Power distribution between SDSG elements in winter

На основании этих результатов можно сделать вывод о том, что минимальный уровень себестоимости электроэнергии достигается при величине срока окупаемости в 12-15 лет. Дальнейшее его увеличение не ведет к существенному снижению себестоимости.

\section{Вероятностный метод анализа надежности автономной СДСГ}

При планировании и проектировании СДСГ важный аспект - оценка надежности их функционирования, требующая сложного моделирования, поскольку необходимо учитывать как технические отказы, так и частичные отказы ФЭП при изменении солнечной радиации. Из-за

$$
-49-
$$


высокой стохастичности генерации солнечной энергии по сравнению с генерацией традиционных источников энергии точная оценка надежности систем генерации, использующих возобновляемые источники энергии, является сложной задачей.

Необходимо произвести расчет надежности автономной СДСГ (см. рис. 1) с учетом стохастического характера солнечной радиации и влияния отказов элементов на работу СДСГ.

Большой объем данных о солнечной радиации затрудняет выполнение практических исследований по оценке надежности. В связи с этим полученные актинометрические данные для конкретного местоположения СДСГ необходимо сгруппировать путем объединения близко лежащих значений солнечной радиации по месяцам года и получения их среднего значения. Такой подход позволяет получить модель солнечной радиации с несколькими состояниями $T_{1}$, $T_{2}, T_{3}, \ldots, T_{p}(p=1,2,3 \ldots l)$.

Для каждого из рассматриваемых состояний $\left(T_{1}, T_{2}, T_{3}, \ldots, T_{p}\right)$ назначаются ремонтные и эксплуатационные режимы работы установки $j=0,1, \ldots, m$, отличающиеся составом и вероятностью повреждения оборудования. Все элементы СДСГ получают номера $i=1,2, \ldots, n$. Последствия отказов элементов установки в различных режимах проявляются как аварии с определенной степенью нарушения работоспособности СДСГ: потеря ФЭП, ДГ, банка АБ, инвертора, конвертора и т. д. Все возможные аварии получают свой код $k=1,2,3 \ldots l$.

Для каждого состояния $T_{p}$ необходимо сформировать матрицу логических связей, фиксирующую, какие отказы к какой аварии приводят в каждом из режимов. Таким образом, можно сформировать массив конъюнкций $\{j \cap i\}$, а затем идентифицировать каждую конъюнкцию как аварию $k$-го вида. Формирование массива конъюнкций $\{j \cap i\}$ осуществляется компьютерной программой, в которой для идентификации аварий используется топологический метод проверки связности узлов схемы электрических соединений СДСГ. При этом схема представляется в виде графа, где узлами являются ДГ, ФЭП, АБ, сборные шины 0,4 кВ и отходящие линии, а ветвями - электрическая связь между ними. Топологический анализ заключается в определении отключившихся узлов и идентификации их вида при наложении отказов узлов и отключений ветвей на режим коммутации схемы в нормальных условиях работы и при производстве ремонтов узлов и ветвей.

Вывод расчетных выражений для частоты и длительности аварий основан на последовательном применении формулы полной вероятности при рассмотрении множества возможных конъюнкций. Вероятность аварии $k$-го вида в $j$-м режиме связана с вероятностью отказа какоголибо $i$-го элемента из $n$ формулой полной вероятности, о. е.,

$$
P_{k j}=\sum_{i=0}^{n} P(j i) P(k / j i),
$$

где $P(i j)$ - вероятность отказа $i$-го элемента в $j$-м режиме; $P(k / j j)$ - вероятность возникновения аварии $k$-го вида при условии отказа $i$-го элемента в $j$-м режиме.

Расчет частоты аварий $k$-го вида в каждом состоянии $T_{p}$ осуществляется по формуле, 1 /год,

$$
\begin{aligned}
& \Lambda\left(k_{\text {о.п }} / k_{\mathrm{в.p}}\right)=\sum_{j=0}^{m} \sum_{i=1}^{n} q_{i} \lambda_{i} L(j, i, k), \\
& L(j, i, k)=\left\{\begin{array}{r}
1, \text { если } j \bigcap i=k \\
0, \text { если } j \bigcap i \neq k
\end{array}\right. \\
& -50-
\end{aligned}
$$


где $q_{j}$ - относительная длительность $j$-го режима; $\lambda_{i}$ - частота отказов $i$-го элемента схемы; $L-$ условная вероятность, являющаяся логической функцией для $k$-й аварии (о. п. - оперативные переключения; в. р. - восстановительный ремонт).

Среднее время восстановления нормального режима работы СДСГ после аварии $k$-го вида определяется по выражению, год,

$$
\tau\left(k_{\mathrm{B} . \mathrm{p}}\right)=\frac{1}{\Lambda\left(k_{\mathrm{B} . \mathrm{p}}\right)}\left(\sum_{j=0}^{m} \sum_{i=0}^{n} q_{i} \lambda_{i} \tau(j, i, k) L(j, i, k)\right),
$$

где $\tau(j, i, k)$ - время восстановления нормального функционирования установки после аварии $k$-го вида в $j$-м режиме при отказе $i$-го элемента.

Среднее значение аварийной недовыработки энергии установкой в течение длительности состояния $T_{p}$ вычисляется по формуле, кВт·ч,

$$
\Delta W_{C \not Y}^{(T)}=T_{p}\left(\sum_{k}^{l} \Lambda\left(k_{\mathrm{B} . \mathrm{p}}\right)\left[\tau\left(k_{\mathrm{B} . \mathrm{p}}\right)-\tau\left(k_{\mathrm{o.II}}\right)\right] \Delta N\left(k_{\mathrm{B} . \mathrm{p}}\right)+\sum_{k}^{l} \Lambda\left(k_{\mathrm{o} . \mathrm{II}}\right) \tau\left(k_{\mathrm{o.II}}\right) \Delta N\left(k_{\mathrm{o} . \mathrm{II}}\right)\right)
$$

где $\Delta N(k)$ - величины аварийных снижений мощности СДСГ при авариях $k$-го вида с длительностью их существования $\tau(k)$.

Снижение мощности, вызванное потерей ДГ, равно его номинальной мощности. Среднее снижение мощности, к которому приводит отказ ФЭП, оценивается для каждого состояния $\mathrm{T}_{p}$, кВт:

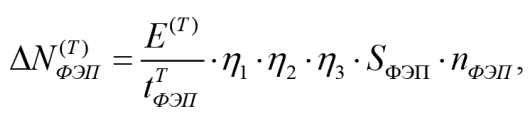

где $E^{(T)}$ - усредненное для каждого состояния $T_{p}$ суточное удельное значение падающей солнечной радиации, кВт•ч/м²; $\eta_{1}-$ КПД ФЭП, о. е.; $\eta_{2}-$ КПД инвертора, о. е.; $\eta_{3}-$ КПД конвертора,

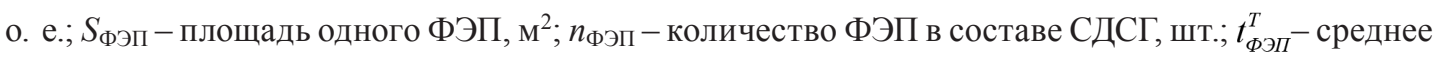
число часов работы ФЭП в сутки для каждого состояния $T_{p}$, зависящее от долготы дня.

Среднее снижение мощности, кВт, вызванное отказом АБ, определяется по выражению

$$
\Delta N_{A B}^{(T)}=\frac{W_{A B}}{t_{A B}^{T}}
$$

где $W_{A D}$ - энергия, запасенная АБ, кВт·ч; $t_{A D}^{T}$ - среднее время непрерывного разряда батареи в течение суток для каждого состояния $T_{p}$, ч.

Показатели надежности элементов СДСГ и данные о солнечной радиации в местоположении рассматриваемого населенного пункта приведены в табл. 2, 3.

Результаты расчета надежности рассмотренной СДСГ (см. рис. 1) приведены в табл. 4. Суммарная недовыработка электроэнергии СДСГ за год составила 485,9 кВт·ч. Для реализации предложенного метода разработана компьютерная программа в среде Matlab. На рис. 6 представлен интерфейс с развернутым окном ввода данных.

Коды аварий: 1 - потеря ДГ; 2 - потеря ФЭП; 3 - потеря АБ; 4 - потеря ДГ и ФЭП; 5 - потеря ДГ и АБ; 6 - потеря ФЭП и АБ; 7 - полное погашение СДСГ. 
Таблица 2. Показатели надежности элементов СДСГ

Table 2. Indicators of reliability of SDSG elements

\begin{tabular}{|l|c|c|c|}
\hline \multicolumn{1}{|c|}{ Элемент } & Номер элемента в схеме & $\lambda, 1 /$ год & $\tau$, год \\
\hline \hline ДГУ & 1 & $1,0 \cdot 10^{-2}$ & $1,0 \cdot 10^{-2}$ \\
\hline ФЭП & 2 & $2,28 \cdot 10^{-2}$ & $6,67 \cdot 10^{-2}$ \\
\hline Блок АБ & 3 & $2,0 \cdot 10^{-3}$ & $1,0 \cdot 10^{-3}$ \\
\hline Инвертор & 5,6 & $5,0 \cdot 10^{-2}$ & $2,0 \cdot 10^{-4}$ \\
\hline Конвертор & 4 & $5,0 \cdot 10^{-2}$ & $2,0 \cdot 10^{-4}$ \\
\hline Шина & 7 & 0,166 & $5,71 \cdot 10^{-4}$ \\
\hline $\begin{array}{l}\text { Автоматический } \\
\text { выключатель }\end{array}$ & $8,9,10$ & $5,0 \cdot 10^{-2}$ & $4,57 \cdot 10^{-4}$ \\
\hline
\end{tabular}

Таблица 3. Данные о солнечной радиации в районе населенного пункта

Table 3. Data on solar radiation in the area of the settlement

\begin{tabular}{|c|c|c|c|c|c|}
\hline Месяц & $E, \kappa \mathrm{B \top} \cdot ч / \mathrm{M}^{2}$ & $E_{\mathrm{ycp}}, \kappa \mathrm{BT} \cdot ч / \mathrm{M}^{2}$ & $\mathrm{~T}_{\mathrm{p}}$ & $\begin{array}{c}\text { Длительность периода } \\
\mathrm{T}_{\mathrm{p}}, \text { дней (ч) }\end{array}$ & $\begin{array}{c}\text { Средняя мощность } \\
\text { ФЭП, кВт }\end{array}$ \\
\hline Январь & 1,25 & \multirow{3}{*}{1,17} & \multirow{3}{*}{$\mathrm{T}_{5}$} & \multirow{3}{*}{92 (2208) } & \multirow{3}{*}{0,853} \\
\hline Ноябрь & 1,34 & & & & \\
\hline Декабрь & 0,92 & & & & \\
\hline Февраль & 2,22 & \multirow{2}{*}{2,25} & \multirow{2}{*}{$\mathrm{T}_{4}$} & \multirow{2}{*}{59 (1416) } & \multirow{2}{*}{1,67} \\
\hline Октябрь & 2,27 & & & & \\
\hline Март & 3,63 & \multirow{2}{*}{3,55} & \multirow{2}{*}{$\mathrm{T}_{3}$} & \multirow{2}{*}{$61(1464)$} & \multirow{2}{*}{2,63} \\
\hline Сентябрь & 3,48 & & & & \\
\hline Апрель & 4,53 & \multirow{2}{*}{4,69} & \multirow{2}{*}{$\mathrm{T}_{2}$} & \multirow{2}{*}{$61(1464)$} & \multirow{2}{*}{3,48} \\
\hline Август & 4,86 & & & & \\
\hline Май & 5,28 & \multirow{3}{*}{5,45} & \multirow{3}{*}{$\mathrm{T}_{1}$} & \multirow{3}{*}{92 (2208) } & \multirow{3}{*}{4,04} \\
\hline Июнь & 5,58 & & & & \\
\hline Июль & 5,49 & & & & \\
\hline
\end{tabular}

Таблица 4. Результаты расчета надежности СДСГ

Table 4. Results of calculating the reliability of SDSG

\begin{tabular}{|l|c|c|c|c|c|c|c|}
\hline \multicolumn{1}{|c|}{ Код аварии } & 1 & 2 & 3 & 4 & 5 & 6 & 7 \\
\hline$\Lambda(k), 1 /$ год & 0,0755 & 0,2174 & 0,1283 & $1,449 \cdot 10^{-4}$ & $1,843 \cdot 10^{-5}$ & $2,086 \cdot 10^{-4}$ & 0,2090 \\
\hline$\tau(k)$, год & 0,0406 & 0,1795 & 0,0068 & 0,0351 & 0,0060 & 0,0066 & 0,0113 \\
\hline$\Delta W_{p}(k)$, кВт·ч & 214,8 & 34,5 & 18,3 & 0,362 & 0,01 & 0,03 & 217,9 \\
\hline
\end{tabular}




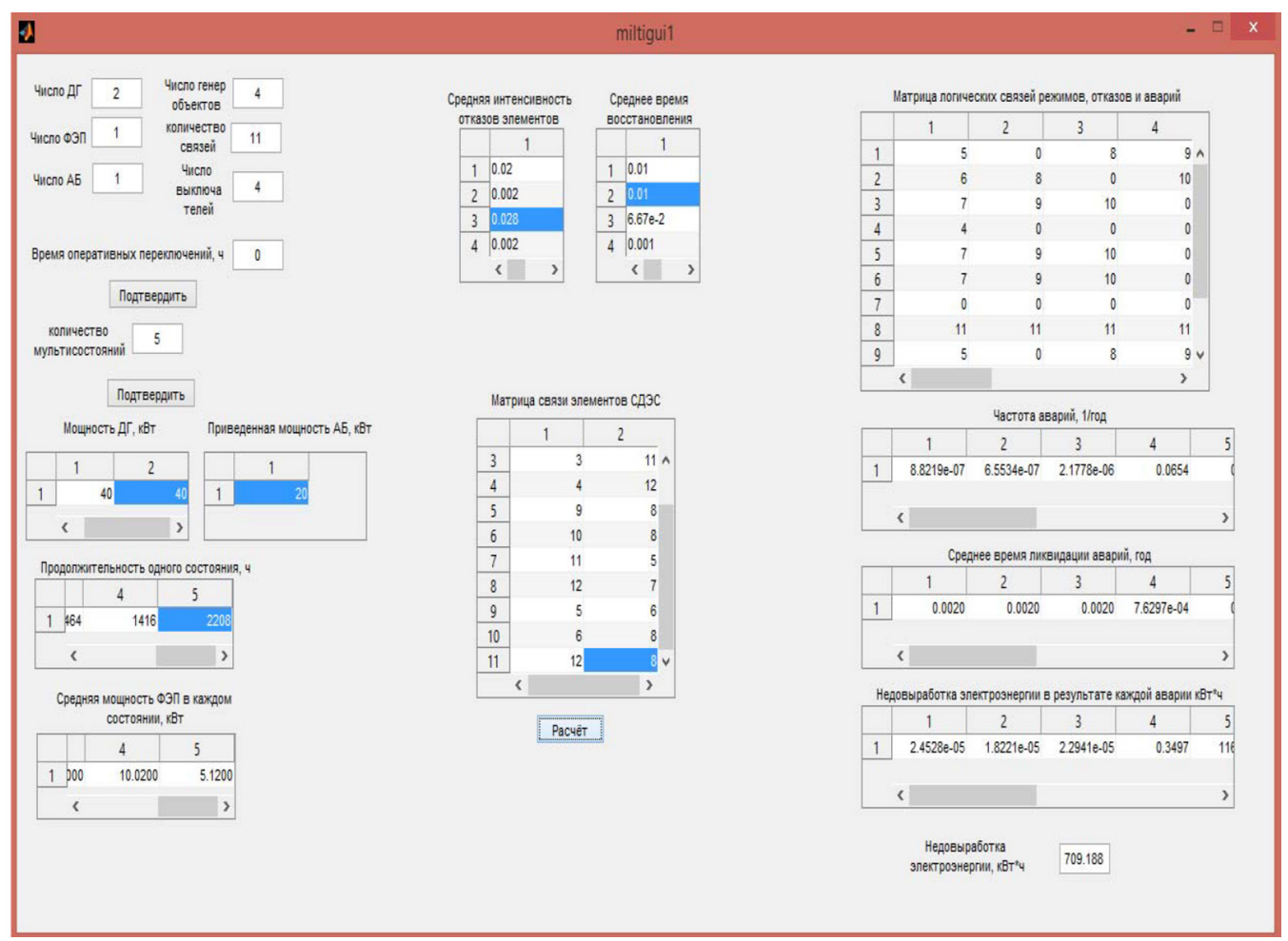

Рис. 6. Интерфейс компьютерной программы

Fig. 6. Computer program interface

\section{Заключение}

Разработана методика оптимизации состава автономных солнечно-дизельных электростанций, использующая данные о выработке электроэнергии ФЭП с учетом суточной цикличности и сезонной изменчивости интенсивности солнечного излучения. Это позволяет обеспечить снижение расхода дизельного топлива и, как следствие, снижение издержек при выработке электроэнергии.

Результаты оптимизации СДСГ для села в Хакасии показали, что установка ФЭП на неподвижную опору с наклоном, соответствующим широте местности, обеспечивает наиболее полное использование солнечной энергии для покрытия суточной потребности населенного пункта в электроэнергии.

На основе предложенного вероятностного метода выполнен анализ надежности полученного оптимального состава СДСГ.

\section{Список литературы / References}

[1] Дмитриенко В.Н., Лукутин Б.В. Выбор мощности генерирующего оборудования автономной солнечно-дизельной электростанции мегаваттного класса. Фундаментальные исследования, 2015, 4, 61-66. [Dmitrienko V.N, Lukutin B. V. The Choice of capacity generation equipment autonomous solar-diesel plant megawatt class, Journal of Basic Research, 2015, 4, 61-66 (in Russian)] 
[2] Гапоненко, А.М., Каграманова А. А. Оптимизация состава комплекса возобновляемых источников энергии с использованием кластерного подхода и теории случайных процессов. Научный журнал КубГАУ, 2016, 116(02), 1-16 [Gaponenko A. M., Kagramanova A. A. Optimization of the composition of the complex of renewable energy sources with the use of cluster approach and the theory of random processes. Scientific Journal of Kuban State Agrarian University, 2016, 116(02), 1-16 (in Russian)]

[3] Rout K., Sahu J.K. Various optimization techniques of hybrid renewable energy systems for power generation: a review. International Research Journal of Engineering and Technology, 2018, 5(7), 1173-1176

[4] Bhandari B., Lee Kyung-Tae, Lee Gil-Yong, Cho Young-Man, Ahn Sung-Hoon. Optimization of hybrid renewable energy power systems: a review. International Journal of Precision Engineering and Manufacturing-Green Technology, 2015, 2(1), 99-112

[5] Lopez R.D,. Agustin J. L.B. Design and control strategies of PV-diesel systems using genetic algorithms. Solar Energy, 2005, 79(1), 33-46

[6] Lilienthal P.D., Lambert T. W., Gilman P. Computer modeling of renewable power systems. Encyclopedia of Energy, 2004, 1, 633-647

[7] Minna Ranjeva, Anil K. Kulkarni. Design optimization of a hybrid, small, decentralized power plant for remote / rural areas. Energy Procedia, 2012, 20, 258-270

[8] Kornelakis A, Marinakis Y. Contribution for optimal sizing of grid-connected PV-systems using PSO. Renewable Energy, 2010, 35(6), 1333-1341

[9] Shi Y., Eberhart R. A modified particle swarm optimizer. Proceedings of the IEEE International Conference on Evolutionary Computation, Piscataway, NJ, 1998, 69-73

[10] Тремясов В.А., Кенден К.В. Фотоэлектрические и гидроэнергетические установки в системах автономного электроснабжения. Красноярск, Сиб. федер. ун-т, 2017. 208 с. [Tremasov V.A., Kenden K.V. Photovoltaic and hydropower plants in autonomous power supply systems. Krasnoyarsk, Sib. Fed. University, 2017, 208 p. (in Russian)] 